\title{
A QUASI-NEWTON ALGORITHM BASED ON A REDUCED MODEL FOR FLUID-STRUCTURE INTERACTION PROBLEMS IN BLOOD FLOWS*
}

\author{
JeAn-FrÉdéric Gerbeau ${ }^{1}$ And Marina Vidrascu ${ }^{1}$
}

\begin{abstract}
We propose a quasi-Newton algorithm for solving fluid-structure interaction problems. The basic idea of the method is to build an approximate tangent operator which is cost effective and which takes into account the so-called added mass effect. Various test cases show that the method allows a significant reduction of the computational effort compared to relaxed fixed point algorithms. We present 2D and 3D fluid-structure simulations performed either with a simple 1D structure model or with shells in large displacements.
\end{abstract}

Mathematics Subject Classification. 65M60, 74K25, 76D05, 76Z05.

\section{INTRODUCTION}

This work deals with the numerical simulations of the mechanical interaction between the blood and the wall of large arteries. This subject is of considerable interest as shown by numerous studies in bioengineering and applied mathematics (see e.g. $[2,12,22,23,27,29]$ ). From the numerical viewpoint, it is now currently accepted that these fluid-structure problems differ from those encountered in other domains (aeroelasticity for example). The interaction between arterial walls and blood flows belongs to the category of problems for which the numerical stability strongly depends on the accuracy of the resolution of the fluid-structure coupling at each time step (see [11]). In other words, a precise energy balance must be obtained at each time step and consequently the coupling has to be solved by implicit schemes. The resulting nonlinear problem is generally solved by sub-structuring methods based on fixed point algorithms (see e.g. $[5,11,15,20]$ ), direct procedures are also used in some cases (see [28,31]). Adaptive relaxation (see [15-17]) allows a considerable improvement with respect to standard relaxed fixed point methods. Yet, fluid-structure simulations for blood flows are still very expensive with these methods. In order to decrease the computational cost, it is thus natural to think of Newton methods. For example, a block-Newton method has been proposed in [13,14]. In these studies the action of the tangent operators is approximated by finite differences. In [7], the tangent operator is computed exactly, but the method has not yet been implemented.

We propose in this article a quasi-Newton method (which has been briefly presented in [9] in 2D). Basically, the idea of our approach is to approximate the tangent operator of the nonlinear fluid-structure problem by a much simpler operator. The difficulty is to design a pertinent reduced model ("reduced" being understood here in the sense of "with a reduced complexity"). Some reduced fluid-structure models are well-known in the

Keywords and phrases. Fluid-structure interaction, quasi-Newton algorithm, added mass effect, blood flows.

* This work has been partially supported by the Research Training Network "Mathematical Modelling of the Cardiovascular System" (HaeMOdel), contract HPRN-CT-2002-00270 of the European Community.

1 INRIA Rocquencourt, BP 105, 78153 Le Chesnay Cedex, France. e-mail: Jean-Frederic.Gerbeau@inria.fr 


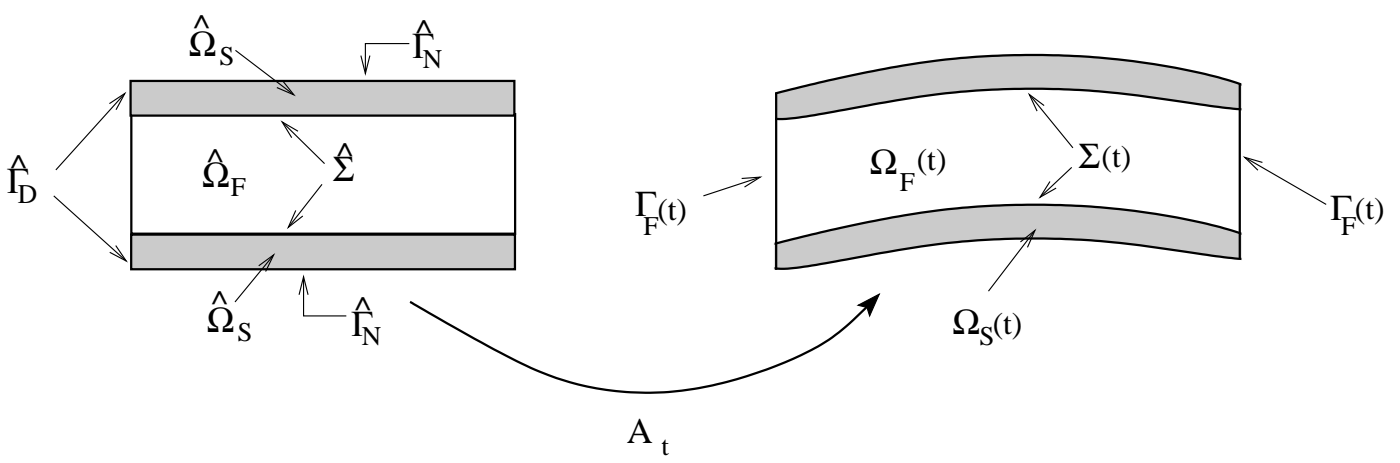

Figure 1. An example of the reference and current configurations.

context of haemodynamics, for example 1D nonlinear hyperbolic models (see e.g. $[8,21,30]$ ). Nevertheless, it is difficult to exploit their results within 3D simulations because of the difference of nature of the variables: velocity, pressure, displacement in 3D versus flux, mean pressure, section area in 1D. Moreover these 1D models generally assume a cylindrical geometry, which is too restrictive.

One of the contributions of the present work is to propose a model which uses the same variables as the 3D fluid-structure problem, which does not require particular geometries and which is a "reasonable" approximation of the original problem. From a physical viewpoint, it is "a priori reasonable" in the sense that it captures the added mass effect which is an important mechanical feature of the fluid-structure problems encountered in blood flows. From a numerical viewpoint, it is "a posteriori reasonable" since it provides a pertinent approximation of the tangent operator of the nonlinear problem, at least in the test cases which have been considered.

The article is organized as follows. Section 2 is devoted to the general formulation of the fluid-structure problem. Section 3 describes the reduced model and Section 4 presents the numerical methods used in this study, in particular our algorithm is presented in Section 4.4. The performance of the algorithms are assessed in Section 5 where the results obtained in 2D and 3D numerical simulations are described.

\section{Formulation of the Fluid-STRUCtURE PROBLEM}

\subsection{The governing continuum equations}

In order to tackle each problem in its natural setting, we make the standard choice (see e.g. [6, 11]) to consider the structure in a pure Lagrangian framework, and the fluid in an Arbitrary Lagrangian Eulerian (ALE) formulation. More precisely, denoting by $\Omega_{F}(t)$ (resp. $\Omega_{S}(t)$ ) the fluid (resp. structure) domain at time $t$, we introduce an application $\mathcal{A}_{t}$ defined on a reference configuration $\hat{\Omega}_{F}$ (resp. $\hat{\Omega}_{S}$ ) which maps any point $\hat{x} \in \hat{\Omega}_{F}\left(\right.$ resp. $\left.\hat{\Omega}_{S}\right)$ onto $x=\mathcal{A}_{t}(\hat{x}) \in \Omega_{F}(t)$ (resp. $\Omega_{S}(t)$ ) (Fig. 1). We introduce the following notations:

$$
\boldsymbol{w}=\frac{\partial \mathcal{A}_{t}}{\partial t}, \quad \boldsymbol{F}=\frac{\partial \mathcal{A}_{t}}{\partial \hat{x}}, \quad J=\operatorname{det} \boldsymbol{F} .
$$

In the structure, $\mathcal{A}_{t}$ is such that $\mathcal{A}_{t}(\hat{x})$ corresponds to the current position of the material point located in $\hat{x}$ at time $t=0$. Thus, in particular, $\boldsymbol{F}$ denotes the deformation gradient and $\boldsymbol{w}$ is the velocity of the structure. In the fluid, the mapping $\mathcal{A}_{t}$ is just required to satisfy kinematic constraints on the boundary. In the sequel, $\Sigma(t)$ denotes the fluid-structure interface, namely $\partial \Omega_{F}(t) \cap \partial \Omega_{S}(t)$. We denote by $\Gamma_{F}(t)$ the portion of the fluid boundary that is not shared with the boundary of structure, $\Gamma_{F}(t)=\partial \Omega_{F}(t) \backslash \Sigma(t)$. The boundary $\partial \hat{\Omega}_{S}$ of the structure is partitioned in $\hat{\Gamma}_{D}, \hat{\Gamma}_{N}$ and $\hat{\Sigma}$. We denote by $\boldsymbol{n}$ the outward normal on the fluid boundary, and by $\boldsymbol{n}_{S}$ the outward normal on the structure boundary. We denote by $\boldsymbol{u}$ the velocity and $p$ the pressure in the fluid, and by $\boldsymbol{d}$ (resp. $\boldsymbol{w}$ ) the displacement (resp. the velocity) in the structure. 
Fluid problem

The equations for the fluid problem are:

$$
\left\{\begin{aligned}
\rho_{F}\left(\left.\frac{\partial \boldsymbol{u}}{\partial t}\right|_{\hat{x}}+(\boldsymbol{u}-\boldsymbol{w}) \cdot \boldsymbol{\nabla u}\right) & =\operatorname{div}(2 \mu \boldsymbol{\epsilon}(\boldsymbol{u}))-\nabla p, & & \text { in } \Omega_{F}(t), \\
\operatorname{div} \boldsymbol{u} & =0, & & \text { in } \Omega_{F}(t), \\
\boldsymbol{u}(x, t) & =\frac{\partial \boldsymbol{d}}{\partial t}\left(\mathcal{A}_{t}^{-1}(x), t\right), & & \text { on } \Sigma(t), \\
\boldsymbol{\sigma}_{F} \cdot \boldsymbol{n} & =\boldsymbol{g}, & & \text { on } \Gamma_{F}(t),
\end{aligned}\right.
$$

where $\boldsymbol{\epsilon}(\boldsymbol{u})=\left(\boldsymbol{\nabla} \boldsymbol{u}+\boldsymbol{\nabla} \boldsymbol{u}^{T}\right) / 2$ is the strain rate tensor, $\boldsymbol{\sigma}_{F}=-p \mathbf{1}+2 \mu \boldsymbol{\epsilon}(\boldsymbol{u})$ the Cauchy stress tensor in the fluid, $\rho_{F}$ is the density of the fluid and $\mu$ its dynamic viscosity.

Structure problem

The equation of the structure can be written as:

$$
\left\{\begin{aligned}
\rho_{S} \frac{\partial^{2} \boldsymbol{d}}{\partial t^{2}}-\operatorname{div} \hat{x}(\hat{\boldsymbol{T}}) & =0 & & \text { in } \hat{\Omega}_{S} \\
\hat{\boldsymbol{T}} \cdot \hat{\boldsymbol{n}}_{S} & =J \hat{\boldsymbol{\sigma}}_{F} \cdot \boldsymbol{F}^{-T} \hat{\boldsymbol{n}}_{S} & & \text { on } \hat{\Sigma} \\
\boldsymbol{d} & =0 & & \text { on } \hat{\Gamma}_{D} \\
\hat{\boldsymbol{T}} \cdot \hat{\boldsymbol{n}}_{S} & =0 & & \text { on } \hat{\Gamma}_{N}
\end{aligned}\right.
$$

where $\hat{\boldsymbol{T}}$ is the first Piola-Kirchoff stress tensor. The structure is supposed to be an hyperelastic material, thus the stress tensor $\hat{\boldsymbol{T}}$ is given by the derivative of an internal stored energy function $\mathcal{W}(\boldsymbol{F})$ (see [10]). The choice of the internal stored energy will not change the setting of the fluid-structure problem. It will be then possible to choose the most appropriate model depending on the simulation. The actual structure models which have been used for this work will be briefly described in Section 2.3.

\subsection{Weak formulation}

We introduce the following function spaces:

$$
\begin{gathered}
\hat{V}=\left(H^{1}\left(\hat{\Omega}_{F}\right)\right)^{3}, \quad V=\left\{\hat{\boldsymbol{v}} \circ \mathcal{A}_{t}^{-1}, \hat{\boldsymbol{v}} \in \hat{V}\right\}, \\
\hat{V}_{0}=\left\{\hat{\boldsymbol{v}} \in \hat{V},\left.\hat{\boldsymbol{v}}\right|_{\hat{\Sigma}}=0\right\}, \quad V_{0}=\left\{\hat{\boldsymbol{v}} \circ \mathcal{A}_{t}^{-1}, \hat{\boldsymbol{v}} \in \hat{V}_{0}\right\}, \\
\hat{M}=L^{2}\left(\hat{\Omega}_{F}\right), \quad M=\left\{\hat{q} \circ \mathcal{A}_{t}^{-1}, \hat{q} \in \hat{M}\right\}, \\
X=\left\{\boldsymbol{d} \in\left(H^{1}\left(\hat{\Omega}_{S}\right)\right)^{3},\left.\boldsymbol{d}\right|_{\hat{\Gamma}_{D}}=0\right\} .
\end{gathered}
$$

The variational fluid problem reads: find $(\boldsymbol{u}(t), p(t)) \in V \times M$ such that for all $(\boldsymbol{v}, q) \in V_{0} \times M$,

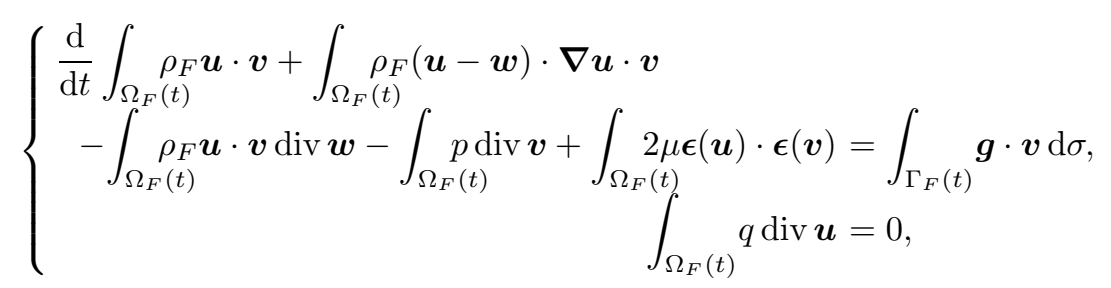

and

$$
\left.\int_{0}^{t} \boldsymbol{u}\right|_{\Sigma}(s) \mathrm{d} s=\left.\boldsymbol{d}\right|_{\hat{\Sigma}}(t)-\left.\boldsymbol{d}\right|_{\hat{\Sigma}}(0)
$$

In the fluid domain, we simply define $\boldsymbol{w}$ by an harmonic extension of the boundary velocity:

$$
-\triangle \boldsymbol{w}=0
$$


with

$$
\left.\boldsymbol{w}\right|_{\Sigma(t)}=\left.\boldsymbol{u}\right|_{\Sigma(t)}
$$

For the structure, we look for $\boldsymbol{d}(t) \in X$, such that for all $\hat{\boldsymbol{\varphi}} \in X$,

$$
\int_{\hat{\Omega}_{S}} \rho_{S} \frac{\partial^{2} \boldsymbol{d}}{\partial t^{2}} \cdot \hat{\boldsymbol{\varphi}} \mathrm{d} \hat{x}+a_{S}(\boldsymbol{d}, \hat{\boldsymbol{\varphi}})=<\boldsymbol{f}_{\Sigma}, \hat{\boldsymbol{\varphi}}>
$$

with

$$
<\boldsymbol{f}_{\Sigma}, \hat{\boldsymbol{\varphi}}>=\int_{\Sigma(t)}(p \boldsymbol{n}-2 \mu \boldsymbol{\epsilon}(\boldsymbol{u}) \cdot \boldsymbol{n}) \cdot \boldsymbol{\varphi} \mathrm{d} \sigma .
$$

The operator $a_{S}$ is in general nonlinear and can be written for example in terms of the free energy function (see e.g. [10]).

\subsection{The structural models}

\subsubsection{A shell model in large displacements}

A general structural model in blood flows for complex realistic geometries has to be three-dimensional. As the wall of the blood vessels is thin, it is convenient to use shell elements. The model used hereafter can handle large displacements, the elements used are MITC4 "General Shell Elements" (see [1,4]).

A major interest of the "General Shell Elements" is that they are based on a general 3D variational formulation. In particular, standard 3D constitutive laws are used. The transverse stress is null and a kinematical constraint makes this model compatible with a Reissner-Mindlin shell model. Hereafter we will use a generalized Hooke law for which the internal stored energy on the non-deformed configuration is given by:

$$
\mathcal{W}(\boldsymbol{d})=\frac{1}{2} \int_{\hat{\Omega}_{S}}\left[C^{\alpha \beta \lambda \mu} e_{\alpha \beta}(\boldsymbol{d}) e_{\lambda \mu}(\boldsymbol{d})+D^{\alpha \lambda} e_{\alpha z}(\boldsymbol{d}) e_{\lambda z}(\boldsymbol{d})\right],
$$

where $\boldsymbol{e}=\left(e_{i j}\right)$ denotes the nonlinear Green-Lagrange deformation tensor. In equation (9), the Greek symbols varying from 1 to 2 are used for the tangential components to the surface, and $z$ is the transverse direction. In addition we have:

$$
\begin{gathered}
C^{\alpha \beta \lambda \mu}=\frac{E}{2(1+\nu)}\left(g^{\alpha \lambda} g^{\beta \mu}+g^{\alpha \mu} g^{\beta \lambda}+\frac{2 \nu}{1-\nu} g^{\alpha \beta} g^{\lambda \mu}\right), \\
D^{\alpha \lambda}=\frac{8 E}{t^{2}(1+\nu)} g^{\alpha \lambda},
\end{gathered}
$$

where $E$ is the Young modulus, $\nu$ the Poisson ratio and $g^{\alpha \lambda}$ the contravariant components of the metric tensor.

The finite element used in our computations is the MITC4 element. The geometry is fully defined by the position of the nodes $\boldsymbol{x}^{(i)}$ on the mid-surface, the unit outward normal $\boldsymbol{a}_{3}^{(i)}$ at the surface in each node and the corresponding thickness $t^{(i)}$ (see Fig. 2). The position of any point in the structure is given by:

$$
\boldsymbol{x}=\sum_{i} \lambda_{i}(r, s)\left(\boldsymbol{x}^{(i)}+z \frac{t^{(i)}}{2} \boldsymbol{a}_{3}^{(i)}\right)
$$

where $\lambda_{i}(r, s)$ are the standard Q1 Lagrange shape functions in 2D. The displacement of this point is then given by:

$$
\boldsymbol{d}=\sum_{i} \lambda_{i}(r, s)\left(\boldsymbol{d}^{(i)}+z \frac{t^{(i)}}{2} \boldsymbol{\eta}^{(i)}\right)
$$

where $\boldsymbol{d}^{(i)}$ is the displacement of the node $i$, while $\boldsymbol{\eta}^{(i)}$ corresponds to the rotation of the unit vector $\boldsymbol{a}_{3}^{(i)}$ and verifies, for the small displacements, the constraint:

$$
\boldsymbol{\eta}^{(i)} \cdot \boldsymbol{a}_{3}^{(i)}=0
$$




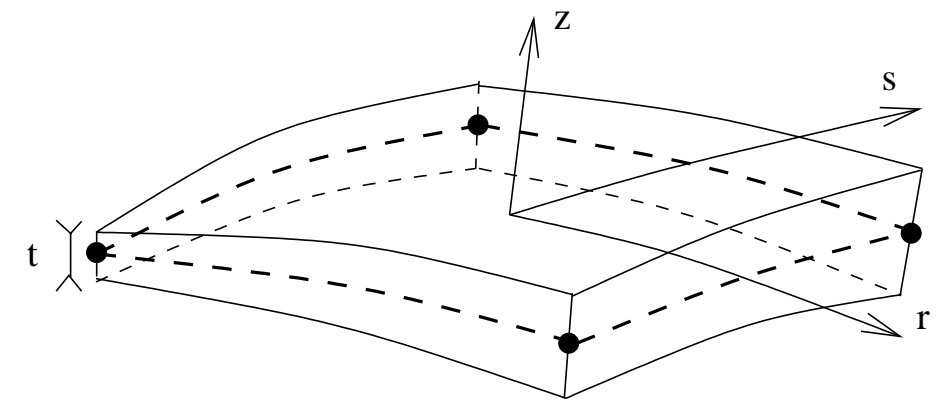

FiguRE 2. General shell element (Q1) with local coordinates system.

The MITC4 finite element has thus 5 degrees of freedom per node (the three components of the displacement and the two parameters which define the variation of the unit vector). This element is robust and does not appear significantly affected by locking (see [4]). This desirable feature is obtained by using a particular interpolation strategy for the various components of the strain tensor (we refer to [1] for further details).

\subsubsection{A generalized string model}

In order to test the coupling algorithms, it will be convenient to use also a simplified structural model which is derived from the theory of linear elasticity, for a cylindrical tube with small thickness, under the assumptions of membrane deformations. This model has been used in many works devoted to blood flows (see for example [25]). The reference configuration is a cylindrical surface of axis $0 z$ and whose base is a circle of radius $R_{0}$, the radial displacement $d_{r}=d_{r}(z, t)$ is given by

$$
\rho_{w} h \frac{\partial^{2} d_{r}}{\partial t^{2}}-k G h \frac{\partial^{2} d_{r}}{\partial z^{2}}+\frac{E h}{1-\nu^{2}} \frac{d_{r}}{R_{0}^{2}}-\gamma \frac{\partial^{3} d_{r}}{\partial z^{2} \partial t}=f_{\Sigma},
$$

where $h$ is the wall thickness, $k$ is the so-called Timoshenko shear correction factor, $G$ the shear modulus, $E$ the Young modulus, $\nu$ the Poisson ratio, $\rho_{w}$ the wall density and $\gamma$ a viscoelastic parameter. The longitudinal and angular displacement are neglected. This simple model will be used in Section 5 for the 2D simulations.

\section{Formulation of a ReDUCED MOdel}

\subsection{General case}

The reduced fluid model that we now present relies on the following basic statement: an important mechanism in fluid-structure problems in arteries is the added mass effect, and this mechanism can be reasonably captured with a simple linear inviscid model for the fluid. More precisely, we suppose that fluid domain $\bar{\Omega}_{F}$ is fixed, and that the pressure and the velocity satisfy:

$$
\left\{\begin{aligned}
\rho_{F} \partial_{t} \boldsymbol{u}+\boldsymbol{\nabla} p & =0 & & \text { in } \bar{\Omega}_{F} \\
\boldsymbol{u} & =\partial_{t} \boldsymbol{d} & & \text { on } \bar{\Sigma} \\
-p \boldsymbol{n} & =\boldsymbol{g} & & \text { on } \bar{\Gamma}_{F}
\end{aligned}\right.
$$

Eliminating $\boldsymbol{u}$, the simplified fluid problem becomes:

$$
\left\{\begin{aligned}
-\triangle p & =0 & & \text { in } \bar{\Omega}_{F} \\
\frac{\partial p}{\partial \boldsymbol{n}} & =-\rho_{F} \frac{\partial^{2} \boldsymbol{d}}{\partial t^{2}} \cdot \boldsymbol{n} & & \text { on } \bar{\Sigma} \\
p & =-\boldsymbol{g} \cdot \boldsymbol{n} & & \text { on } \bar{\Gamma}_{F} .
\end{aligned}\right.
$$


This simplified model is not well-suited for the direct resolution of the fluid-structure problems that we consider here. Indeed, in blood flows, the viscous and the nonlinear advection effects are known to be important and the domain experiences large displacements. Nevertheless, we will show in the sequel that (15) can be used to evaluate efficiently an approximate tangent operator for the genuine fluid-structure problem.

\subsection{The special case of the generalized string model}

For the reader who is not familiar with the notion of added mass, we provide the equation describing the interaction between the simplified fluid equation described above and the generalized string model (14). We first introduce the following notation: let $q$ be given on $\bar{\Sigma}$, we denote by $\mathcal{M}_{\mathcal{A}} q$ the trace on $\bar{\Sigma}$ of $p$, the solution to:

$$
\left\{\begin{aligned}
-\triangle p & =0 & & \text { in } \bar{\Omega}_{F} \\
\frac{\partial p}{\partial \boldsymbol{n}} & =q & & \text { on } \bar{\Sigma} \\
p & =-\boldsymbol{g} \cdot \boldsymbol{n} & & \text { on } \bar{\Gamma}_{F}
\end{aligned}\right.
$$

where $\boldsymbol{g}$ is the same as in (3). Note that $\mathcal{M}_{\mathcal{A}}$ is the inverse of the standard Steklov-Poincaré operator (see [26] for example).

In the simplified model, the fluid being inviscid, the force acting on the structure is solely due to the pressure. Moreover, taking into account the geometrical assumptions made in the definition of the generalized string model, it is clear that $\boldsymbol{d} \cdot \boldsymbol{n}=d_{r}$. Thus, we have:

$$
f_{\Sigma}=p_{\Sigma}=-\rho_{F} \mathcal{M}_{\mathcal{A}} \frac{\partial^{2} d_{r}}{\partial t^{2}}
$$

Finally, equations (14) and (15) give:

$$
\left(\rho_{w} h+\rho_{F} \mathcal{M}_{\mathcal{A}}\right) \frac{\partial^{2} d_{r}}{\partial t^{2}}-k G h \frac{\partial^{2} d_{r}}{\partial z^{2}}+\frac{E h}{1-\nu^{2}} \frac{d_{r}}{R_{0}^{2}}-\gamma \frac{\partial^{3} d_{r}}{\partial z^{2} \partial t}=0 .
$$

Under this form, it clearly appears that the fluid acts as an "added mass" on the structure. In blood flows, this effect is important since the density of the wall and the blood are close. This explains in particular the necessity of implicit coupling algorithms for such problems. A complete discussion on the mass added effect in more general cases is given in [18].

Remark 3.1. Equation (16) constitutes a 1D fluid-structure model, which, to the best of our knowledge, has never been considered in the context of haemodynamics. Compared to the standard 1D models in blood flows, dissipation by viscous effect in the fluid and nonlinearity have been lost. But here, the behaviour of the structure is described more precisely than with the algebraic relations between the area of a section and the pressure commonly used in standard 1D models.

\section{NUMERICAL METHODS}

\subsection{Time-stepping algorithm}

Suppose that the displacement $\boldsymbol{d}^{n+1}$ of the structure is known at time $t^{n+1}$. Then, the velocity $\boldsymbol{w}^{n+1}$ of the fluid domain is computed by:

$$
\left\{\begin{aligned}
\int_{\Omega_{F}^{n+1}} \boldsymbol{\nabla} \boldsymbol{w}^{n+1} \cdot \nabla \boldsymbol{v} & =0 \\
\left.\boldsymbol{w}^{n+1}\right|_{\Sigma^{n+1}} & =\frac{\left.\boldsymbol{d}^{n+1}\right|_{\hat{\Sigma}}-\left.\boldsymbol{d}^{n}\right|_{\hat{\Sigma}}}{\delta t}
\end{aligned}\right.
$$


which is a variational formulation of equations (5) and (6). Note that it is also possible to compute the integrals in (17) in the reference domain. The velocity $\boldsymbol{u}^{n+1}$ and the pressure $p^{n+1}$ are obtained by solving (3) via an implicit Euler scheme:

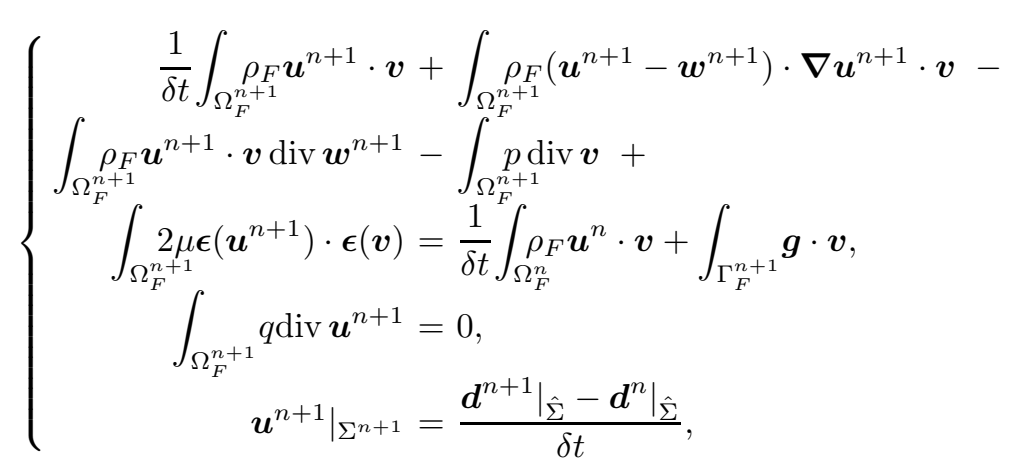

the domain $\Omega_{F}^{n+1}$ being defined by:

$$
\Omega_{F}^{n+1}=\Omega_{F}^{n}+\delta t \boldsymbol{w}^{n+1}
$$

Next, the forcing term $\boldsymbol{f}_{\Sigma}^{n+1}$ is deduced by computing the variational residual of the fluid problem on the fluidstructure interface ( $c f$. [11]). In the sequel, denoting by $\boldsymbol{d}_{\Sigma}$ the displacement of the fluid-structure interface, all this step will be concisely written as:

$$
\boldsymbol{f}_{\Sigma}^{n+1}=\mathcal{F}\left(\boldsymbol{d}_{\Sigma}^{n+1}\right) \text {. }
$$

Now, suppose that the forcing term $\boldsymbol{f}_{\Sigma}^{n+1}$ is known at time $t^{n+1}$. Then, the displacement $\boldsymbol{d}^{n+1}$ and the velocity $\boldsymbol{w}^{n+1}$ of the structure are computed by solving (7) with a mid-point scheme:

$$
\begin{aligned}
\int_{\hat{\Omega}_{S}} \rho_{S} \frac{\boldsymbol{w}^{n+1}-\boldsymbol{w}^{n}}{\delta t} \cdot \hat{\boldsymbol{\varphi}} \mathrm{d} \hat{x}+\frac{1}{2} a_{S}\left(\boldsymbol{d}^{n}, \hat{\boldsymbol{\varphi}}\right)+\frac{1}{2} a_{S}\left(\boldsymbol{d}^{n+1}, \hat{\boldsymbol{\varphi}}\right) & =<\boldsymbol{f}_{\Sigma}^{n+1}, \hat{\boldsymbol{\varphi}}> \\
\frac{\boldsymbol{d}^{n+1}-\boldsymbol{d}^{n}}{\delta t} & =\frac{\boldsymbol{w}^{n+1}+\boldsymbol{w}^{n}}{2}
\end{aligned}
$$

from which is deduced in particular the displacement $\boldsymbol{d}_{\Sigma}^{n+1}$ of the fluid-structure interface. This step will be formally summarized by the formula:

$$
\boldsymbol{d}_{\Sigma}^{n+1}=\mathcal{S}\left(\boldsymbol{f}_{\Sigma}^{n+1}\right)
$$

With these notations, the fluid-structure coupling is achieved at time $t^{n+1}$ by solving the following nonlinear problem on the interface:

$$
\boldsymbol{d}_{\Sigma}^{n+1}=\mathcal{S} \circ \mathcal{F}\left(\boldsymbol{d}_{\Sigma}^{n+1}\right)
$$

In many practical situations (e.g. aeroelasticity), it is not necessary to solve (21), and much time-saving algorithms may be used ( $c f .[24])$. Nevertheless, in fluid-structure problems arising in blood flows, it seems that (21) must be solved very accurately at each time step in order to ensure the overall stability of the simulation. We are now going to present several approaches to solve (21).

\subsection{Fixed point strategy}

A natural way to solve (21) is to use a fixed point algorithm. For this choice, numerical experiments show that, in the cases of interest, such a method fails if an appropriate relaxation strategy is not adopted. The algorithms usually proposed in the literature have the following form:

i) Prediction of the position of the interface:

$$
\boldsymbol{d}_{\Sigma, 0}^{n+1}=\boldsymbol{d}_{\Sigma}^{n}+\frac{3 \delta t}{2} \boldsymbol{w}_{\Sigma}^{n}-\frac{\delta t}{2} \boldsymbol{w}_{\Sigma}^{n-1}, k=0 .
$$


ii) Solution of the coupled fluid-structure problem:

$$
\tilde{\boldsymbol{d}}_{\Sigma, k+1}^{n+1}=\mathcal{S} \circ \mathcal{F}\left(\boldsymbol{d}_{\Sigma, k}^{n+1}\right) .
$$

iii) Correction of the position of the interface:

$$
\boldsymbol{d}_{\Sigma, k+1}^{n+1}=\omega_{k} \tilde{\boldsymbol{d}}_{\Sigma, k+1}^{n+1}+\left(1-\omega_{k}\right) \boldsymbol{d}_{\Sigma, k}^{n+1}
$$

iv) While a stopping test is not satisfied, go to (ii).

The relaxation parameter may be chosen either constant over the whole simulation [20], or dependent either on the time step $n$ [19] or on the sub-step $k$. In this last case, let us mention two possibilities. The first one (proposed in [11] and also used in [15])) is a domain decomposition approach which consists in reformulating the fixed point algorithm as a preconditioned steepest descent method applied to an interface problem involving the Steklov-Poincaré operators of the fluid and the structure. The second possibility suggested in $[15,17]$, is to use a variant of the Aitken acceleration formula, which can be for example written as:

$$
\omega_{k}=\frac{\left(\boldsymbol{d}_{k}-\boldsymbol{d}_{k-1}\right) \cdot\left(\boldsymbol{d}_{k}-\mathcal{S} \circ \mathcal{F}\left(\boldsymbol{d}_{k}\right)-\boldsymbol{d}_{k-1}+\mathcal{S} \circ \mathcal{F}\left(\boldsymbol{d}_{k-1}\right)\right)}{\left|\boldsymbol{d}_{k}-\mathcal{S} \circ \mathcal{F}\left(\boldsymbol{d}_{k}\right)-\boldsymbol{d}_{k-1}+\mathcal{S} \circ \mathcal{F}\left(\boldsymbol{d}_{k-1}\right)\right|^{2}} .
$$

Empirically, we observed that the above cited variant of adaptive relaxation strategies give similar results. As noticed in [15], the Aitken method gives in general slightly better results. Therefore in the sequel, we will compare the results obtained with the new algorithm to those obtained with the Aitken method.

Remark 4.1. In the practical evaluation of $\mathcal{F}\left(\boldsymbol{d}_{\Sigma, k}^{n+1}\right)$, the equations (17) and (18) are in fact solved sequentially: (17) is solved on $\Omega_{F, k}^{n+1}$, which allows to compute the domain $\Omega_{F, k+1}^{n+1}$ on which (18) is then solved.

\subsection{Quasi-Newton algorithms}

Another possible strategy to solve (21) is to use a quasi-Newton method. Denoting by $\mathcal{R}$ the operator $\mathcal{I}-\mathcal{S} \circ \mathcal{F}$, a quasi-Newton algorithm requires the following steps:

i) Prediction of the position of the interface:

$$
\boldsymbol{d}_{\Sigma, 0}^{n+1}=\boldsymbol{d}_{\Sigma}^{n}+\frac{3 \delta t}{2} \boldsymbol{w}_{\Sigma}^{n}-\frac{\delta t}{2} \boldsymbol{w}_{\Sigma}^{n-1}, k=0 .
$$

ii) Solution of an approximate tangent problem:

$$
\tilde{\mathcal{R}}^{\prime}\left(\boldsymbol{d}_{\Sigma, k}^{n+1}\right) \delta \boldsymbol{d}_{\Sigma, k+1}=-\mathcal{R}\left(\boldsymbol{d}_{\Sigma, k}^{n+1}\right) .
$$

iii) Correction of the position of the interface:

$$
\boldsymbol{d}_{\Sigma, k+1}^{n+1}=\boldsymbol{d}_{\Sigma, k}^{n+1}+\lambda_{k} \delta \boldsymbol{d}_{\Sigma, k+1} .
$$

iv) While a stopping test is not true, go to (ii),

where $\tilde{\mathcal{R}}^{\prime}$ is an approximation of the tangent operator, and $\lambda_{k}$ is a coefficient determined by a suitable line search algorithm.

One standard possibility (see e.g. [3]) is to perform an approximate solution of the linear problem of step (ii) with an iterative method (GMRES for example) and, inside this iterative method, evaluate each matrix-vector product $\tilde{\mathcal{R}}^{\prime}\left(\boldsymbol{d}_{\Sigma, k}^{n+1}\right) \boldsymbol{z}$ using a finite difference approximation of first order:

$$
\tilde{\mathcal{R}}^{\prime}\left(\boldsymbol{d}_{\Sigma, k}^{n+1}\right) \boldsymbol{z} \approx \frac{\mathcal{R}\left(\boldsymbol{d}_{\Sigma, k}^{n+1}+\alpha \boldsymbol{z}\right)-\mathcal{R}\left(\boldsymbol{d}_{\Sigma, k}^{n+1}\right)}{\alpha}
$$


or second order:

$$
\tilde{\mathcal{R}}^{\prime}\left(\boldsymbol{d}_{\Sigma, k}^{n+1}\right) \boldsymbol{z} \approx \frac{\mathcal{R}\left(\boldsymbol{d}_{\Sigma, k}^{n+1}+\alpha \boldsymbol{z}\right)-\mathcal{R}\left(\boldsymbol{d}_{\Sigma, k}^{n+1}-\alpha \boldsymbol{z}\right)}{2 \alpha} .
$$

We will briefly discuss this approach in Remark 5.1. An alternative to this algorithm, proposed in next section, is to approximate the tangent operator using the simple fluid model presented in Section 3.

\subsection{The reduced model based quasi-Newton algorithm}

As already mentioned, the simplified fluid model presented in Section 3.1 is linear. Thus, its tangent operator can be straightforwardly evaluated. Note that independently of the model used, the tangent operator of the structure equations is always available: if the model is linear (Sect. 2.3.2), this operator is straightforward, and if the structure is nonlinear (Sect. 2.3.1), its tangent operator needs to be itself computed for the resolution of the structure problem. Thus, the tangent operator of the fluid-structure problem using the fluid model of Section 3.1 and one of the structure models of Section 2.3, can be easily and efficiently evaluated. This is the basic idea of the method that we propose. With the same formal notations as before, the step (ii) in Section 4.3 becomes:

where $\tilde{\mathcal{F}}$ denotes the reduced fluid model of Section 3.1. To be precise, we now describe our algorithm in details.

Suppose that $\Omega_{F}^{n}, \boldsymbol{u}^{n}, p^{n}, \boldsymbol{d}^{n}$ and $\boldsymbol{w}^{n}$ are known at time $t^{n}$, we compute these variables at time $t^{n+1}$ as follows:

1. Prediction of the position of the interface:

$$
\boldsymbol{d}_{\Sigma, 0}^{n+1}=\boldsymbol{d}_{\Sigma}^{n}+\frac{3 \delta t}{2} \boldsymbol{w}_{\Sigma}^{n}-\frac{\delta t}{2} \boldsymbol{w}_{\Sigma}^{n-1}
$$

set $k=0$.

2. Evaluation of the residual $\mathcal{R}_{k}$ of the genuine fluid-structure problem:

2.1. Compute $\boldsymbol{w}_{k+1}^{n+1}$ in the fluid by solving (17) (where $\boldsymbol{d}^{n+1}$ is of course replaced by $\boldsymbol{d}_{k}^{n+1}$ ).

2.2. Move the fluid mesh: $\Omega_{F, k+1}^{n+1}=\Omega_{F}^{n}+\delta t \boldsymbol{w}_{k+1}^{n+1}$.

2.3. Compute $\left(\boldsymbol{u}_{k+1}^{n+1}, p_{k+1}^{n+1}\right)$ by solving (18) in $\Omega_{F, k+1}^{n+1}$. Evaluate the variational residual of the fluid problem to obtain the force $\boldsymbol{f}_{\Sigma, k+1}^{n+1}$ acting on the structure.

2.4. Compute $\left(\boldsymbol{d}_{k+1}^{n+1}, \boldsymbol{w}_{k+1}^{n+1}\right)$ by solving the structure problem with the force $\boldsymbol{f}_{\Sigma, k+1}^{n+1}$. For a nonlinear structure, this step is performed with a Newton-Raphson algorithm. We denote by $D \mathcal{K}_{k+1}^{n+1}$ the tangent operator of the structure problem which is computed during this step.

2.5. The nonlinear residual is: $\mathcal{R}_{k}=\boldsymbol{d}_{\Sigma, k}^{n+1}-\boldsymbol{d}_{\Sigma, k+1}^{n+1}$.

3. If

$$
\frac{\left|\boldsymbol{d}_{\Sigma, k}^{n+1}-\boldsymbol{d}_{\Sigma, k+1}^{n+1}\right|}{\left|\boldsymbol{d}_{r e f}\right|} \leq \varepsilon
$$

go to step 4. Else:

3.1. Compute $\delta \boldsymbol{d}_{\Sigma, k+1}$ by solving the approximate tangent problem with GMRES:

$$
\mathcal{R}^{\prime} \delta \boldsymbol{d}_{\Sigma, k+1}=-\mathcal{R}_{k} .
$$

The matrix $\mathcal{R}^{\prime}$ is not explicitly computed. The evaluation of the product of $\mathcal{R}^{\prime}$ by a vector $\boldsymbol{z}$ (one time per GMRES iteration) is performed as follows:

(i) Solve:

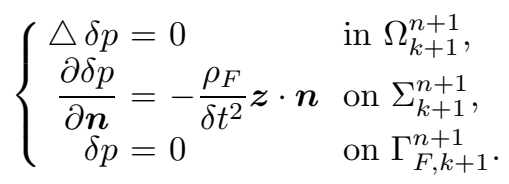


(ii) Define $\delta \boldsymbol{f}$ by

(iii) Solve:

$$
<\delta \boldsymbol{f}, \hat{\boldsymbol{\varphi}}>=\int_{\Sigma_{k+1}^{n+1}} \delta p \boldsymbol{n} \cdot \boldsymbol{\varphi} d \sigma
$$

$$
D \mathcal{K}_{k+1}^{n+1} \delta \boldsymbol{z}=\delta \boldsymbol{f}
$$

(iv) The product $\mathcal{R}^{\prime}$ by $\boldsymbol{z}$ is given by $\boldsymbol{z}-\delta \boldsymbol{z}$.

The GMRES iterations are stopped as soon as the norm of the linear residual is lower than $\varepsilon_{\text {lin }}$ times the norm of the nonlinear residual.

3.2. Compute the new displacement of the interface:

$$
\boldsymbol{d}_{\Sigma, k+1}^{n+1}=\boldsymbol{d}_{\Sigma, k}^{n+1}+\lambda_{k} \delta \boldsymbol{d}_{\Sigma, k+1}
$$

where $\lambda_{k}$ is computed, if necessary (i.e. if the increment with $\lambda_{k}=1$ does not decrease the norm of the residual), by a line search strategy.

3.3. Go to step 2.

4. Go to next time step with $\Omega_{F}^{n+1}=\Omega_{F, k+1}^{n+1}, \boldsymbol{u}^{n+1}=\boldsymbol{u}_{k+1}^{n+1}, p^{n+1}=p_{k+1}^{n+1}, \boldsymbol{d}^{n+1}=\boldsymbol{d}_{k+1}^{n+1}$ and $\boldsymbol{w}^{n+1}=\boldsymbol{w}_{k+1}^{n+1}$.

Remark 4.2. In all the test cases that we performed, the line search algorithm was in fact never activated in step 3.2 (in other words, $\lambda_{k}=1$ always gave a reduction of the residual). This is highly desirable since each iteration of line search requires the expensive evaluation of the fluid-structure residual. This fact confirms the pertinence of the approximate tangent operator that we propose.

Remark 4.3. If formula (23) is used to evaluate the matrix-vector product inside the linear iterative solver (GMRES), then the steps 3.1 (i) to (iv) are replaced by one evaluation of the complete fluid-structure problem, namely one resolution of the Navier-Stokes equation and one resolution of the nonlinear structure problem. If formula (24) is used, then two evaluations of the complete fluid-structure problem are necessary. In our approach, the matrix-vector product only requires the resolution of a scalar Poisson problem (step 3.1 (i)) and a linearized structure problem (step 3.1 (iii)). Moreover, since we use a direct solver for the structure equation, the linearized structure problem was already factorized during step 2.4. The resolution 3.1 (iii) is a forward-backward substitution and is therefore extremely cheap.

Remark 4.4. In step $3,\left|\boldsymbol{d}_{\text {ref }}\right|$ denotes a given reference displacement. Other stopping criteria may be considered, for example replace $\boldsymbol{d}_{r e f}$ by $\boldsymbol{d}_{\Sigma, k+1}^{n+1}$, or involve also the velocity. In all the simulations of blood flows, we use the following values: $\varepsilon=10^{-6},\left|\boldsymbol{d}_{r e f}\right|=1$ and $\varepsilon_{l i n}=10^{-3}$. The value of $\varepsilon_{l i n}$ is almost optimal in the sense that a bigger value leads to divergence whereas a lower one did not improve the convergence of the nonlinear iterations. The value $\varepsilon$ could be a little bit bigger, e.g. about $10^{-5}$, but for $\varepsilon \approx 10^{-4}$ instabilities appear. Notice that for a large value of $\varepsilon$ the coupled fluid-structure problem is in fact not solved in an implicit way.

\section{NumericAl RESULTS}

\subsection{A benchmark test}

This first test case is not related to blood flows: it was proposed in [15] and is used here to validate our codes. A fluid fills a square cavity with a flexible bottom. An oscillating velocity in imposed on the top of the cavity (Fig. 3). The length of the sides of the cavity is 1 and two apertures of length 0.1 are present at the top of the left and right sides. Homogeneous Neumann boundary conditions are imposed on these apertures. The properties of the fluid are $\rho_{F}=1, \mu=0.01$, and those of the structure are $\rho_{S}=500, E=250, h=0.002$. The pulsation of the velocity on the top is $\omega=2 \pi / 5$ and the time step is $\delta t=10^{-1}$. In the original test case of [15], the fluid is $2 \mathrm{D}$ and the structure $1 \mathrm{D}$. Here, we perform a $3 \mathrm{D}$ simulation that mimics the $2 \mathrm{D}$ configuration, the bottom being represented with one layer of nonlinear shell elements. The elevation of three points of the bottom (initial abscissa $0.25,0.5$ and 0.75 ) are represented in Figure 4 . The results are in very good agreements 


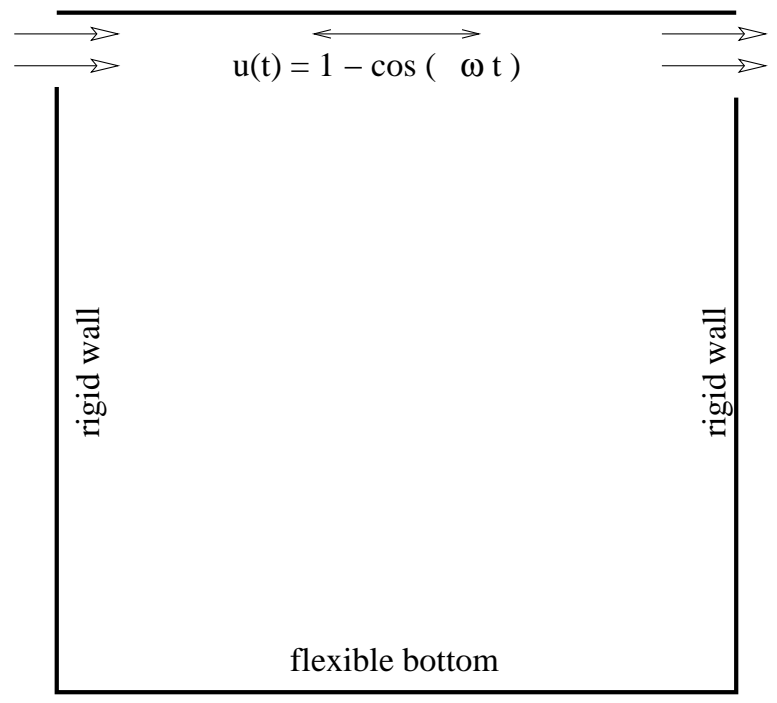

FiguRE 3. Oscillating fluid in a cavity with an elastic bottom.

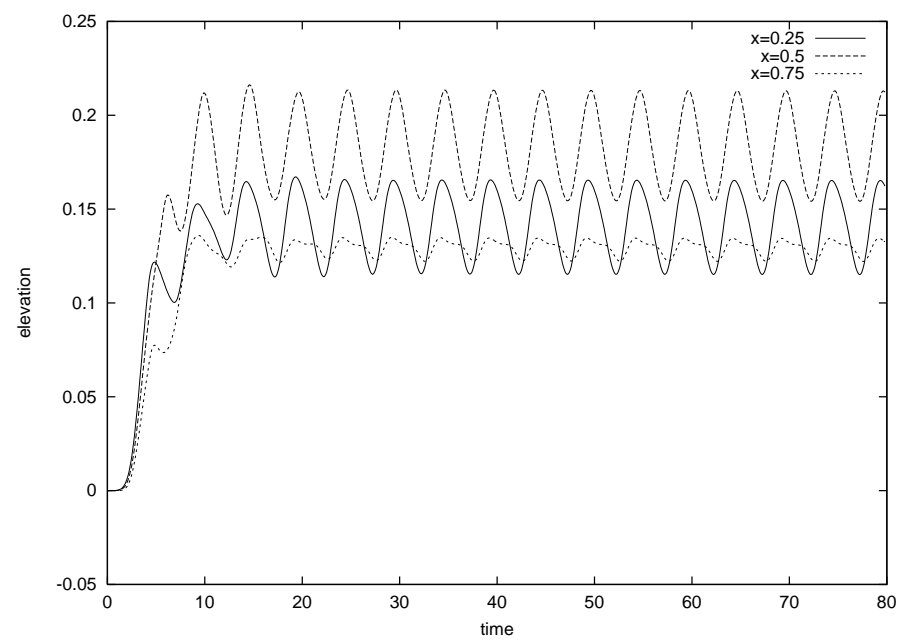

Figure 4. Elevation of three points of the flexible bottom.

with those presented in [15]. Note that in this situation, the convergence is achieved with only 2 or 3 Aitken iterations at each time step. Quasi-Newton methods are therefore useless is this case.

\subsection{Pressure wave in $2 \mathrm{D}$}

We now present the result obtained on the $2 \mathrm{D}$ benchmark proposed in [8]: the domain is a rectangular ( $H=1 \mathrm{~cm}, L=6 \mathrm{~cm}$ ). The structure is described by the generalized string model (Sect. 2.3.2). The fluid is initially at rest and an over pressure of $2 \cdot 10^{4}$ dynes $/ \mathrm{cm}^{2}$ has been imposed at the inlet for 0.005 seconds (Fig. 5 ). For the physical parameters, we chose values that are realistic in the context of blood flow: $\mu=0.035$ poise, $\rho_{F}=1 \mathrm{~g} / \mathrm{cm}^{3}, E=0.75 \times 10^{6}$ dynes $/ \mathrm{cm}^{2}, \nu=0.5, \rho_{S}=1.1 \mathrm{~g} / \mathrm{cm}^{3}$ and $h=0.1 \mathrm{~cm}$. A propagation of the 


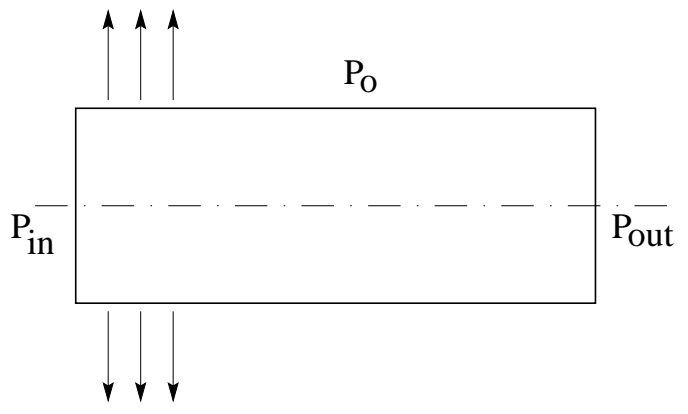

FIGURE 5. Schematic representation the pressure wave test case.
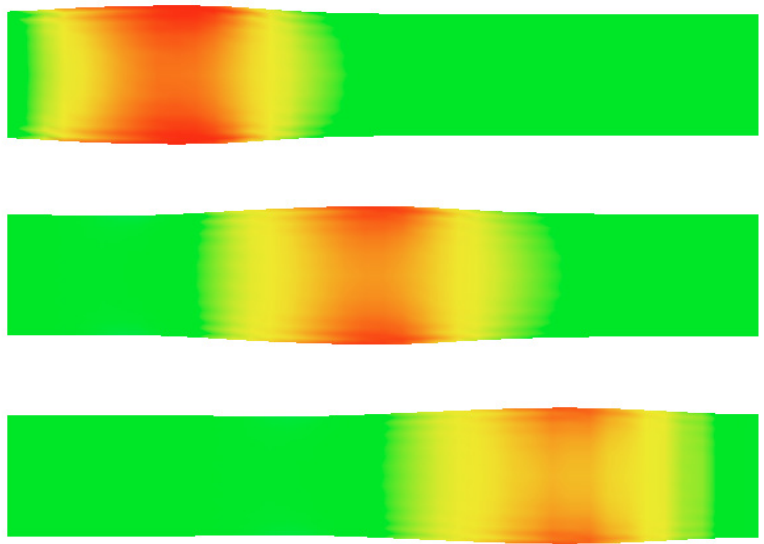

Figure 6. Propagation of a pressure wave in a portion of artery (2D), $t=6,10$ and $14 \mathrm{~ms}$.

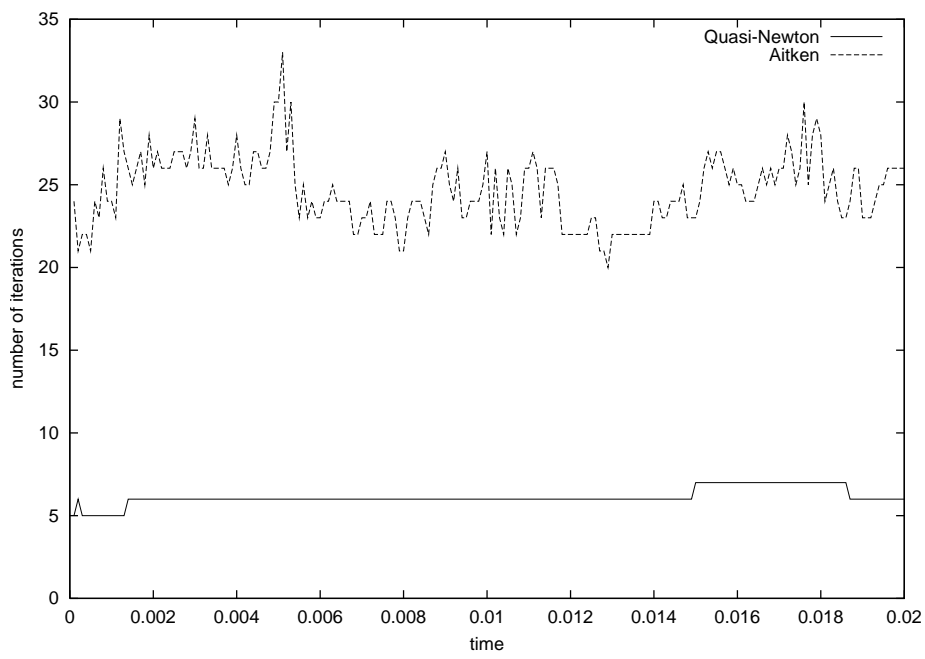

Figure 7. Pressure wave in 2D. Number of iterations. 
pressure wave is observed. For example, Figure 6 shows the pressure at time $t=6,10$ and $14 \mathrm{~ms}$. The obtained results are very similar to those presented in [8]. In Figure 7 are indicated the number of iterations required at each time step to achieve the convergence in problem (21). With a fixed relaxation parameter, fitted by hand, the mean number of iterations is more than one hundred per time step. With the Aitken acceleration method this number is about 24.1 whereas it is about 6.1 with the quasi-Newton algorithm of Section 4.4. In our implementation, the quasi-Newton algorithm was 2.7 times faster than the Aitken method (and more than 10 times faster than the fixed point method with a relaxation parameter fitted by hand).

Remark 5.1. We have also tested the method consisting in evaluating the approximate tangent operator by formula (23) or (24). We did not investigate extensively this approach, so we just make two remarks. First, the choice of the "small" parameter $\alpha$ is - as expected - very sensitive. Second, even when $\alpha$ is perfectly tuned and everything works fine, this approach is still expensive: for example suppose that the convergence is achieved in only 3 Newton iterations, and suppose that each of them requires only 8 GMRES iterations (which corresponds roughly to what has been observed), this yields at least 24 evaluations of the complete fluid-structure operator. Therefore, even in this optimistic situation, we obtain almost the same cost as with the Aitken algorithm.

\subsection{Pressure wave in $3 \mathrm{D}$}

We now consider a 3D version of the previous test case. The parameters are the same as those used in [8]: the domain is a cylinder of radius $R_{0}=0.5 \mathrm{~cm}$ and length $L=5 \mathrm{~cm}$. The structure is a nonlinear shell (Sect. 2.3.1). The fluid is initially at rest and an over pressure of $1.3332 \times 10^{4}$ dynes $/ \mathrm{cm}^{2}(10 \mathrm{mmHg})$ has been imposed at the inlet for 0.005 seconds. The physical parameters are: $\mu=0.03$ poise, $\rho_{F}=1 \mathrm{~g} / \mathrm{cm}^{3}, E=3 \times 10^{6}$ dynes $/ \mathrm{cm}^{2}$, $\nu=0.3, \rho_{S}=1.2 \mathrm{~g} / \mathrm{cm}^{3}$ and $h=0.1 \mathrm{~cm}$. Again, a propagation of the pressure wave is observed (Fig. 8), and the results are similar to those of $[8]$.

With the Aitken acceleration method, the mean number of iterations per time step is about 33.9 whereas it is about 8.9 with the quasi-Newton algorithm (Fig. 9). In our implementation, the quasi-Newton algorithm was 2.4 times faster than the Aitken method.

The same experiment has been performed in a bended tube. The results shown in Figure 10 have been obtained with the quasi-Newton algorithm (with about the same number of iterations as in the previous test case). In this configuration, the fixed point algorithm with Aitken acceleration failed to converge.

For the sake of completeness, let us indicate that each structure resolution in the experiments presented in this section requires about three Newton-Raphson iterations.

\section{Conclusion}

We have presented a method to solve the coupling of the incompressible Navier-Stokes equations on a moving domain with either a generalized wave equation (2D simulations) or a nonlinear shell model (3D simulations). Our algorithm is a quasi-Newton method in which the approximation of the tangent operator is governed by physical considerations. This method offers a possible alternative to the classical relaxed fixed point algorithms commonly used in fluid-structure problems. In the test cases that we have considered it allows a reduction of the CPU time by a factor 2 or 3 compared to the most efficient methods that we know, and by a factor 10 compared to fixed point methods with constant relaxation. Moreover, we have presented a simulation where our algorithm converges and the traditional methods fail. Our approach seems therefore to improve both efficiency and robustness. The results are very promissing, but this algorithm still needs to be validated in other situations, in particular with pulsatile flows, more realistic geometries, and larger displacements. 

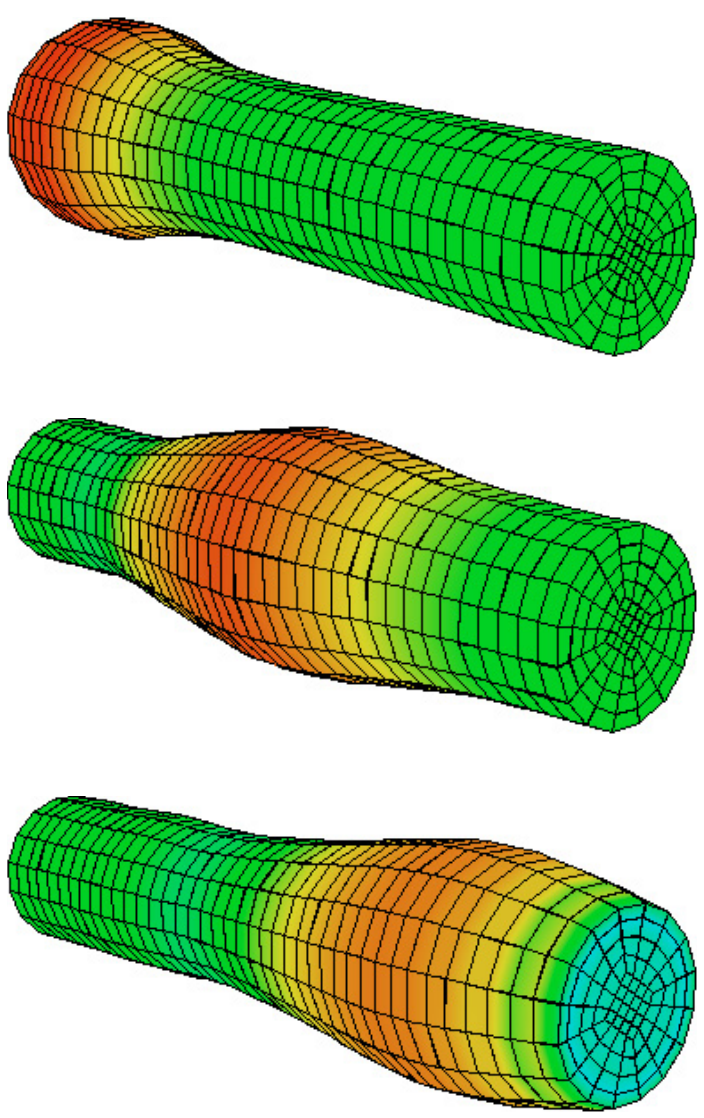

FiguRE 8. Propagation of a pressure wave in a portion of cylinder (3D), $t=4,10$ and $14 \mathrm{~ms}$ (the displacements are magnified by a factor 10).

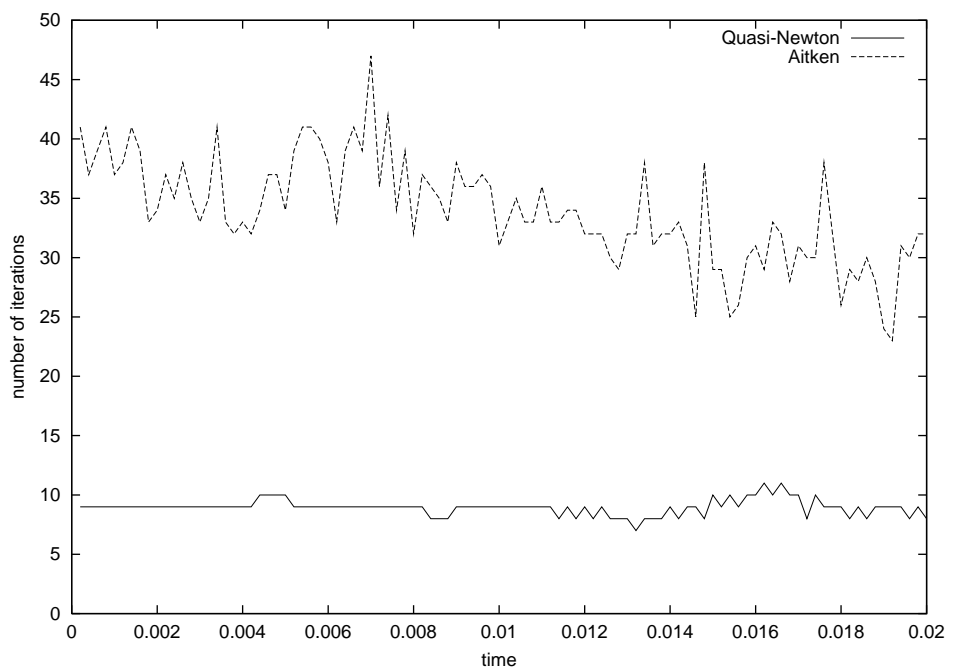

Figure 9. Pressure wave in 3D. Number of iterations. 

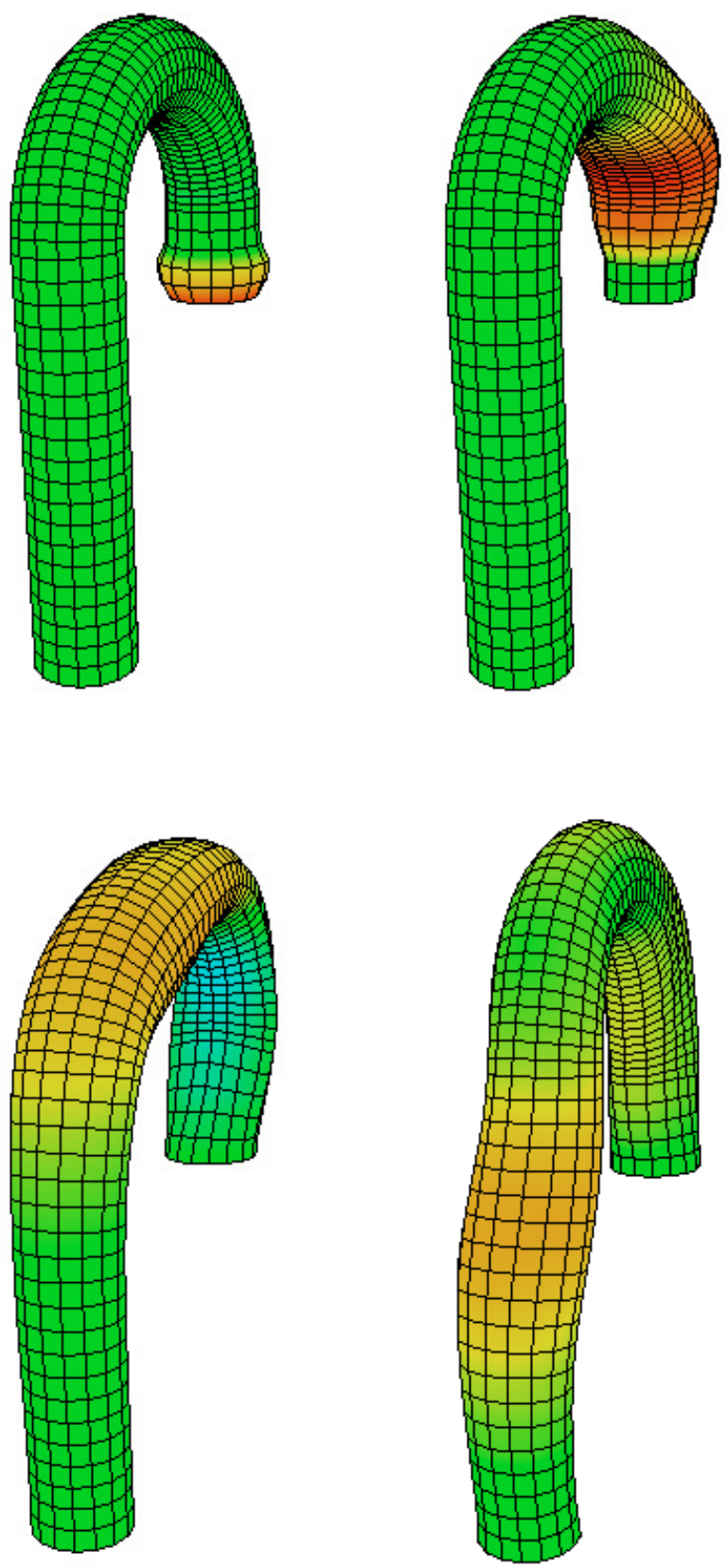

FIGURE 10. Propagation of a pressure wave in a bended tube, $t=1.2,7.2,13.2$ and $19.2 \mathrm{~ms}$ (the displacements are magnified by a factor 10). 


\section{REFERENCES}

[1] K.J. Bathe, Finite Element Procedures. Prentice Hall (1996).

[2] M. Bathe and R.D. Kamm, A fluid-structure interaction finite element analysis of pulsative blood flow through a compliant stenotic artery. J. Biomech. Engng. 121 (1999) 361-369.

[3] P.N. Brown and Y. Saad, Convergence theory of nonlinear Newton-Krylov algorithms. SIAM J. Optim. 4 (1994) $297-330$.

[4] D. Chapelle and K.J. Bathe, The Finite Element Analysis of Shells - Fundamentals. Springer Verlag (2003).

[5] S. Deparis, M.A. Fernández, L. Formaggia and F. Nobile, Acceleration of a fixed point algorithm for fluid-structure interaction using transpiration conditions, in Second MIT Conference on Computational Fluid and Solid Mechanics, Elsevier (2003).

[6] J. Donéa, S. Giuliani and J.P. Halleux, An arbitrary Lagrangian-Eulerian finite element method for transient dynamic fluidstructure interactions. Comp. Meth. Appl. Mech. Engng. (1982) 689-723.

[7] M.A. Fernández and M. Moubachir, An exact block-newton algorithm for the solution of implicit time discretized coupled systems involved in fluid-structure interaction problems, in Second MIT Conference on Computational Fluid and Solid Mechanics, Elsevier (2003).

[8] L. Formaggia, J.-F. Gerbeau, F. Nobile and A. Quarteroni, On the coupling of 3D and 1D Navier-Stokes equations for flow problems in compliant vessels. Comp. Meth. Appl. Mech. Engrg. 191 (2001) 561-582.

[9] J.-F. Gerbeau, A quasi-newton method for a fluid-structure problem arising in blood flows, in Second MIT Conference on Computational Fluid and Solid Mechanics, Elsevier (2003).

[10] P. Le Tallec, Numerical methods for nonlinear three-dimensional elasticity, in Handbook of numerical analysis, Vol. III, NorthHolland (1994) 465-622.

[11] P. Le Tallec and J. Mouro, Fluid structure interaction with large structural displacements. Comput. Meth. Appl. Mech. Engrg. 190 (2001) 3039-3067.

[12] X. Ma, G.C. Lee and S.G. Wu, Numerical simulation for the propagation of nonlinear pulsatile waves in arteries. Transactions of the ASME 114 (1992) 490-496.

[13] H.G. Matthies and J. Steindorf, Partitioned but strongly coupled iteration schemes for nonlinear fluid-structure interaction. preprint, 2000.

[14] H.G. Matthies and J. Steindorf, How to make weak coupling strong, in Computational Fluid and Solid Mechanics, K.J. Bathe Ed., Elsevier (2001) 1317-1319.

[15] D.P. Mok and W.A. Wall, Partitioned analysis schemes for the transient interaction of incompressible flows and nonlinear flexible structures, in Trends in computational structural mechanics CIMNE, K. Schweizerhof, W.A. Wall and K.U. Bletzinger Eds., Barcelona (2001).

[16] D.P. Mok, W.A. Wall and E. Ramm, Partitioned analysis approach for the transient, coupled response of viscous fluids and flexible structures, in Proceedings of the European Conference on Computational Mechanics. ECCM'99, W. Wunderlich Ed., TU Munich (1999).

[17] D.P. Mok, W.A. Wall and E. Ramm, Accelerated iterative substructuring schemes for instationary fluid-structure interaction, in Computational Fluid and Solid Mechanics, K.J. Bathe Ed., Elsevier (2001) 1325-1328.

[18] H. Morand and R. Ohayon, Interactions fluides-structures, Vol. 23 of Recherches en Mathématiques Appliquées. Masson, Paris (1992).

[19] J. Mouro, Interactions fluide structure en grands déplacements. Résolution numérique et application aux composants hydrauliques automobiles. Ph.D. thesis, École Polytechnique, France (1996).

[20] F. Nobile, Numerical approximation of fluid-structure interaction problems with application to haemodynamics. Ph.D. thesis, EPFL, Switzerland (2001).

[21] M.S. Olufsen, Modeling the Arterial System with Reference to an Anesthesia Simulator. Ph.D. thesis, Roskilde University (1998).

[22] K. Perktold and G. Rappitsch, Mathematical modeling of local arterial flow and vessel mechanics, in Computational Methods for Fluid-Structure interaction, J. Crolet and R. Ohayon Eds., Pitman (1994).

[23] K. Perktold and G. Rappitsch, Computer simulation of local blood flow and vessel mechanics in a compliant carotid artery bifurcation model. J. Biomech. 28 (1995) 845-856.

[24] S. Piperno, Explicit/implicit fluid/structure staggered procedures with a structural predictor and fluid subcycling for 2D inviscid aeroelastic simulations. Int. J. Numer. Method Fluid 25 (1997) 1207-1226.

[25] A. Quarteroni, M. Tuveri and A. Veneziani, Computational Vascular Fluid Dynamics: Problems, Models and Methods. Comp. Vis. Sci. 2 (2000) 163-197.

[26] Alfio Quarteroni and Alberto Valli, Domain decomposition methods for partial differential equations. Numerical Mathematics and Scientific Computation. The Clarendon Press Oxford University Press, Oxford Science Publication (1999).

[27] K. Rhee and S.M. Lee, Effects of radial wall motion and flow waveform on the wall shear rate distribution in the divergent vascular graft. Ann. Biomed. Eng. (1998).

[28] S. Rugonyi and K.J. Bathe, On finite element analysis of fluid flows fully coupled with structural interactions. CMES 2 (2001). 
[29] D. Tang, J. Yang, C. Yang and D.N. Ku, A nonlinear axisymmetric model with fluid-wall interactions for steady viscous flow in stenotic elastic tubes. J. Biomech. Engng. 121 (1999) 494-501.

[30] S.A. Urquiza, M.J. Venere, F.M. Clara and R.A. Feijóo, Finite element (one-dimensional) haemodynamic model of the human arterial system, in ECCOMAS, Barcelona (2000).

[31] H. Zhang and K.J. Bathe, Direct and iterative computing of fluid flows fully coupled with structures, in Computational Fluid and Solid Mechanics, K.J. Bathe Ed., Elsevier (2001) 1440-1443.

To access this journal online:

www.edpsciences.org 